\title{
Cytogenetic studies in Dictyophorus griseus (Reiche \& Fairmaire, 1849) (Orthoptera: Pyrgomorphidae) from Cameroon. II. Karyotype
}

\author{
Seino Richard Akwanjoh', Manjeli Yacouba ${ }^{2}$, and Dongmo Tonleu Ingrid ${ }^{1}$ \\ ${ }^{1}$ Department of Animal Biology, Faculty of Science, University of Dschang, Cameroon \\ ${ }^{2}$ Department of Animal Production, Faculty of Agronomy and Agricultural Sciences, University \\ of Dschang, Cameroon
}

\begin{abstract}
The detailed karyotype information (chromosome number, morphology and chromosome lengths) of Dictyophorus griseus (Reiche \& Fairmaire, 1849), a Pyrgomorphidae grasshopper common to the Western Highlands of Cameroon has been described for the first time. The species revealed a karyotype of $2 \mathrm{n} \hat{\gamma}=19 \times \mathrm{X}$ acrocentric chromosomes. Chromosome lengths varied from 8.40 to $2.10 \mu \mathrm{m}$ and the haploid set was $43.87 \mu \mathrm{m}$. The karyotype revealed 2 large, 4 medium and 4 small chromosomes (2LL - 4MM - 4SS). The X chromosome was the longest chromosome in the karyotype.
\end{abstract}

Key words: Dictyophorus griseus, Pyrgomorphidae, karyotype, chromosome length

\section{INTRODUCTION}

Although 'polymorphisms' in karyotypes have been reported in several wild populations of species, the architecture and composition of a species' karyotype is relatively stable (Channaveerappa and Ranganath 1997). It is for this reason that the karyotype is an important asset of a species and serves in the identification of a species. Several papers have been published on the cytogenetics of Pyrgomorphidae but the vast majority of them come from species in the Asian and the Neotropical regions. So far, the karyotypes (chromosome number, sex determination and chromosome morphology) of only four African Pyrgomorphidae grasshoppers (Zonocerus variegatus, Taphronota thaelephora, Pyrgomorpha rugosa and $P$. granulata) have been studied (Oyidi, 1967, Faluyi and Olorode, 1988, Fossey et al., 1989, Seino et al., 2002, 2007). The chromosome numbers in these species range from $2 \mathrm{n} \quad \hat{}=11$ to 20 with karyotypes that are acrocentric or may possess submetacentric chromosomes. The presence of centric fusions and B-chromosomes has resulted in the observed variations in chromosome number and morphology. These reports have revealed a fundamental karyotype of $2 \mathrm{n}{ }^{\hat{1}}=19 \times \mathrm{X}$ acrocentric chromosomes for Pyrgomorphidae grasshoppers (White, 1973; Hewitt, 1979, Santos et al., 1983). In spite of these, the cytogenetics of Cameroonian Pyrgomorphidae is still poorly understood and for 12 of the 14 described species completely unavailable.

To further extend our knowledge of the cytogenetics of the Cameroonian fauna of Pyrgomorphidae, the karyotype (chromosome number and morphology) and chromosome lengths in $D$. griseus were investigated and reported here.

\section{MATERIAL AND METHODS}

In May 2011, ten adult males of $D$. griseus were collected from the village of Angong-Ngie in Momo Division of the North West Region in Cameroon and used for this study. On capture, the insects were injected with $1 \mathrm{ml}$ of $1 \%$ colchicines and allowed for 48 hours before being killed with chloroform fumes and then dissected in insect saline $(0.68 \% \mathrm{NaCl})$ for the testes. The testes obtained were placed in $3: 1$ ethanol: acetic acid fixative and stored in the refrigerator at $4^{\circ} \mathrm{C}$ until used.

Chromosome smears were prepared using the Lactic-Propionic-Orcein squash technique (Seino et al., 2010). These chromosome smears were examined using the Fisher laboratory microscope by first scanning with the 10X objective and nuclei of interest further examined with the high power objective 40X. Chromosome morphology was determined by examining the shapes of chromosome in meiotic Anaphase-I, Metaphase-II and Anaphase-II and classified as per the criteria of Williams and Ogunbiyi, (1995) and Seino et al., (2008). Photographs of mitotic chromosome smears were taken with the Lietz digital photomicroscope using the oil immersion lens, 100X. Sufficient photographs were taken and mitotic metaphases were processed using the Microsoft Office Picture Manager. 
The lengths of individual chromosomes were measured directly from the microscope using ocular and stage micrometers. Each chromosome per mitotic Metaphase cell was measured several times until the measurement obtained did not vary. The ocular divisions obtained were then recorded and converted into micrometers upon calibration with the stage micrometer. Individual chromosome pairs were identified on the basis of length (Stace, 1980).

Relative chromosome length (RCL) is the length of each chromosome expressed as a percentage of the total haploid autosome length in the nucleus (Paris Conference, 1971). This was calculated during this study by adding together the lengths of all the autosomes in one nucleus, then dividing by 2 to obtain the total haploid length since the autosomes were paired. To obtain the RCL, each chromosome length was next divided by the total haploid length and multiplied by 100 to gain a percentage result.

Data on RCL were used to construct graphs to determine size groups. To confirm the size groups, the mean chromosome lengths of the three size groups were subjected to the Duncan's Multiple Range test, (DMRT) (Steel and Torrie, 1981).

\section{RESULTS AND DISCUSSION}

The family of Pyrgomorphidae grasshoppers is known to have a highly conserved karyotype made up of 19 acrocentric chromosomes in male individuals (White, 1973; Hewitt, 1979). In spite of this high level of conservatism, it is known that the karyology of every species is unique to itself and provides an identity to the species (Channaveerappa and Ranganath, 1994). The necessity therefore arose for the investigation and elucidation of the cytogenetics of $D$. griseus whose karyotype is here described for the first time.

Analysis of mitotic Metaphases (Fig. 1) in D. griseus revealed 19 chromosomes. Analysis of cells in Diplotene (Fig. 2) and Metaphase - 1(Fig 3) revealed 9 bivalents and one univalent indicating that the diploid number in this species is $2 n=19{ }^{\lambda} \mathrm{XO}$. Mitotic chromosomes in $D$. griseus were rod-shaped (Fig.1) and centromeres were not distinct in them. The chromosomes in Anaphase -1 (Fig. 4) were made up of two chromatids held at one end and there was repulsion towards the other end conferring a V-shape on the chromosome. In Metaphase -2, the chromosomes were still V- shaped (Fig. 5) but in Anaphase -2 (Fig. 6) the chromatids were separated and the chromosomes appeared I - shaped. As per the criteria of Williams and Ogunbiyi (1995) these chromosomes could be judged to be acrocentric in morphology. From this study, the standard karyotype of $D$. griseus comprises of $2 n=19 \times O$ acrocentric chromosomes which is the same as that described for representatives and basic for the family Pyrgomorphidae. (White, 1973; Hewitt, 1979; Faluyi and Olorode, 1988; Seino et al., 2002).

Actual lengths (micro-meters) and Relative Chromosome Lengths (RCL) obtained during this study are shown in Table 1. Chromosome lengths ranged from 8.40 to $2.10 \mu \mathrm{m}$ and the total haploid set was $43.87 \mu \mathrm{m}$. The Relative Chromosome Lengths (Table 1) were used to construct the graphs in Fig. 7 , which revealed that the chromosomes in $D$. griseus occurred in three size groups of large, medium and small. There were 2pairs of large, 4 pairs of medium and 4 pairs of small chromosome (2LL, 4MM, 4SS) present in the karyotype of this species. The large chromosomes measured 8.40 to $6.83 \mu \mathrm{m}$ with a mean length of $7.62 \mu \mathrm{m}$; the medium chromosomes ranged from 5.34 to $3.5 \mu \mathrm{m}$ with a mean length of $4.66 \mu \mathrm{m}$; while the small chromosomes ranged from 2.89 to $2.10 \mu \mathrm{m}$ with a mean length of $2.5 \mu \mathrm{m}$. The data on mean chromosome lengths per size group (Table 1) were next subjected to the Duncan Multiple Range Test (DMRT) to determine if these means (per size group) were significantly different. The results of this test revealed that the mean length of the large pairs chromosomes $(7.62 \mu \mathrm{m})$ was significantly higher $(P<0.05)$ than that of the medium chromosomes $(4.66 \mu \mathrm{m})$ and the mean length of the medium chromosomes was significantly higher $(P<0.05)$ than that of the small chromosomes $(2.5$ $\mu \mathrm{m})$.

The results obtained in this study permitted us to construct the karyotype of D. griseus (Fig. 7) consisting of 2pairs of large, 4 pairs of medium and 4 pairs of small chromosomes (1LL, 4MM, 4SS). The $\mathrm{X}$ chromosome in $D$. griseus was a large chromosome and measured $8.40 \mu \mathrm{m}$. It was similar to the $X$ chromosome in $Z$. variegatus (Pyrgomorphidae) which was also large and measured 6.77 $\mu \mathrm{m}$ (Faluyi and Olorode, 1988). 
Agric. Biol. J. N. Am., 2012, 3(7): 292-295
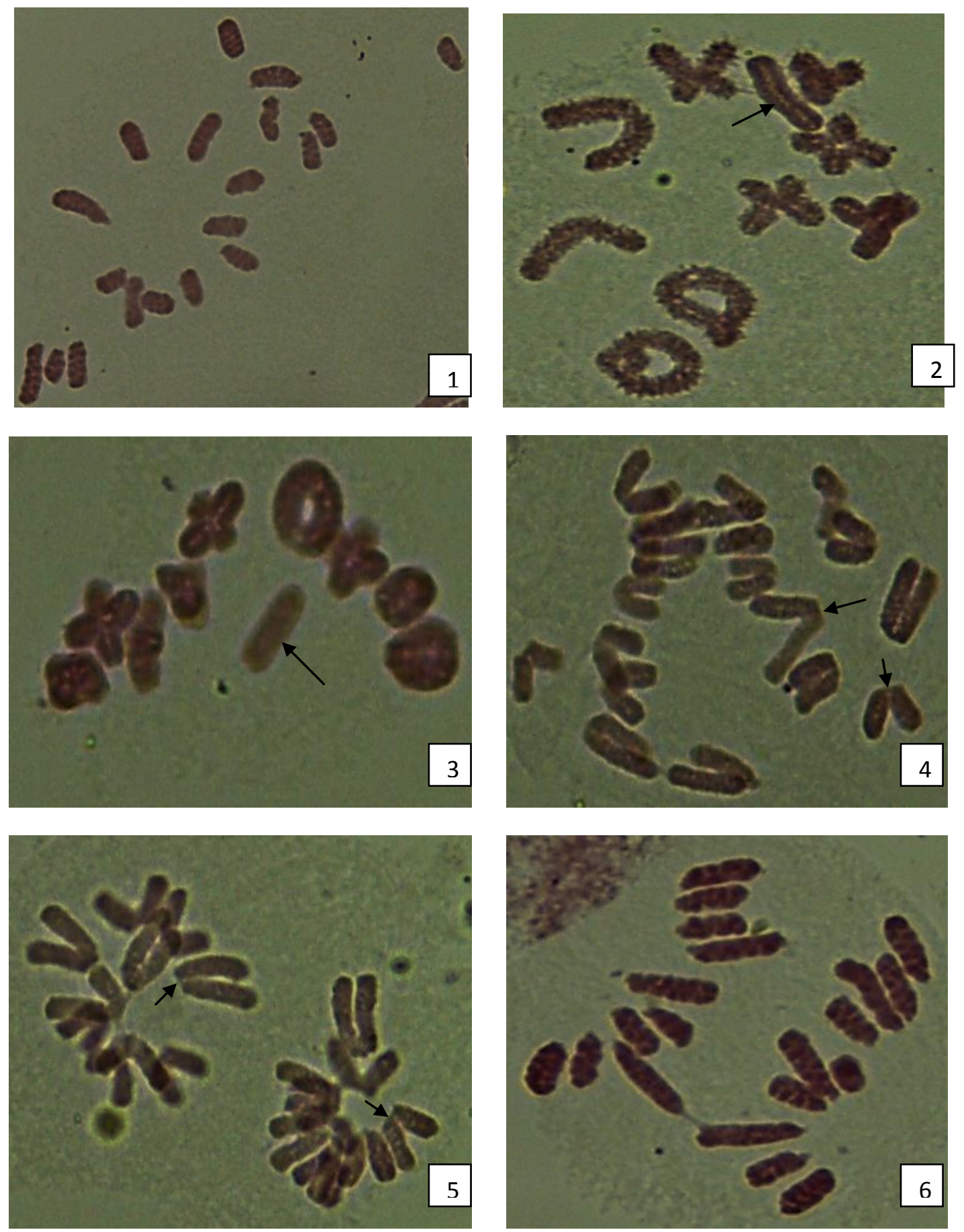

Fig. 1: Mitotic metaphase in $D$. griseus with 19 chromosomes present

Fig. 2: Diplotene in $D$. griseus. Nine bivalents and the univalent $X$ - chromosome (arrowed) are present

Fig. 3: Metaphase -1 in $D$. griseus: Nine bivalents and the univalent $X$ - chromosome (arrowed) are present

Fig. 4: Anaphase -1in D. griseus. The chromosomes are V-shaped with centromere (arrowed) holding the chromatids at one end

Fig. 5: Metaphase -2 in D. griseus. The chromosomes are V-shaped with centromere (arrowed) holding the chromatids at one end

Fig. 6: Anaphase -2 in $D$. griseus. The chromosomes are I - shaped 
Agric. Biol. J. N. Am., 2012, 3(7): 292-295

Table 1: Chromosome lengths, RCL, and chromosome size groups in Dictyophorus griseus

\begin{tabular}{|c|c|c|c|c|c|c|c|c|c|c|}
\hline & \multicolumn{10}{|c|}{ Chromosome pairs } \\
\hline & $\mathbf{X}$ & 1 & 2 & 3 & 4 & 5 & 6 & 7 & 8 & 9 \\
\hline Chromosome length $(\mu \mathrm{m})$ & 8.40 & 6.83 & 5.34 & 5.17 & 4.64 & 3.50 & 2.89 & 2.63 & 2.37 & 2.10 \\
\hline RCL & 19.15 & 15.57 & 12.17 & 11.79 & 10.58 & 7.98 & 6.59 & 5.99 & 5.40 & 4.79 \\
\hline Chromosome size groups & \multicolumn{2}{|c|}{ Large } & \multicolumn{4}{|c|}{ Medium } & \multicolumn{4}{|c|}{ Small } \\
\hline Mean lengths $(\mu \mathrm{m})$ & \multicolumn{2}{|c|}{7.62} & \multicolumn{4}{|c|}{4.66} & \multicolumn{4}{|c|}{2.5} \\
\hline
\end{tabular}

$\mathrm{RCL}=$ Relative Chromosome Length (\% of $\mathrm{n}$ - set)

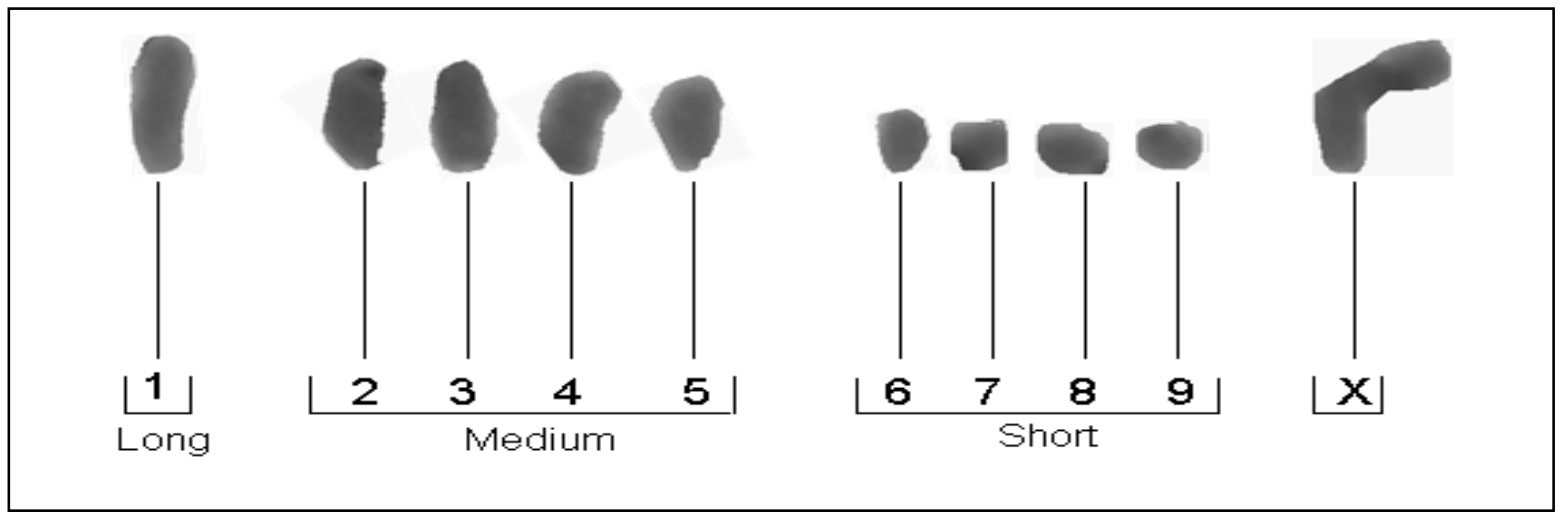

Fig. 7: Karyotype of $D$. griseus. Chromosomes occur in size groups of long, medium and short

\section{REFERENCES}

Channaveerappa H. Ranganath HA. 1994. Karyology of a few species of South Indian Acridids. 1. The chromosomes of Neorthocris acuticeps Bolivar., Sci. Jr. Mysore Univ., 33: $91-96$

Channaveerappa H. Ranganath HA. 1997. Karyology of a few species of South Indian Acridids. II. Male germ line karyotypic instability in Gastrimargus. J. Biosci., 22(3): $367-374$.

Faluyi, JO. and Olorode, O. 1988. Mitotic chromosome studies in the nymphal and adult populations of Zonocerus variegatus L. (Orthoptera: Pyrgomorphidae). Journal of African Zoology, 102 (4): 475 - 480.

Fossey A. Liebenberg H. Jacobs DH. 1989. Karyotype and meiosis studies in three South African Pyrgomorpha species (Orthoptera: Pyrgomorphidae). Genetica, 78: 179 $-183$.

Hewitt GM. 1979. Grasshoppers and Crickets. Animal cytogenetics. Insecta, I. Orthoptera. Vol. 3. Grebruger Borntraeger Ed. Berlin Stuttgart.

Oyidi, O. 1966.Variation and variability in Orthopteran insects. $\mathrm{PhD}$ thesis, University of London.

Paris conference. 1971. Standardization in Human cytogenetics. Birth Defects: Original Article Series 8: 7, 1972 New York: The National Foundation (Reprinted in Cytogenetics 11: 1313 -362 (1972)

Santos JL. Arana P. Giraldez R. 1983. Chromosome Cbanding patterns in Spanish Acridoidea. Genetica, 61: 65 -74 .
Seino, RA. Focho DA. Njukeng FA. 2002. Observations on the meiotic process in the African pest grasshopper Taphronota thaelephora Stal. (Orthoptera: Pyrgomorphidae). J. Acad. Scies. 2 (1): $3-6$.

Seino, RA. Manjeli, Y. Focho, DA. Shambo, DN. 2007. The B chromosome in the meiotic process of the African pest grasshopper Taphronota thaelephora Stal. (Orthoptera: Pyrgomorphidae). International Journal of Biological and Chemical sciences, 1(2): 151 - 157.

Seino RA. Akongnui T. Dongmo NB. Manjeli Y. 2008. Karyotype and meiosis studies in Oxycatantops spissus. (Orthoptera: Acrididae). International Journal of Biological and Chemical Sciences. 2 (2): 168-174.

Seino RA. Akongnui T. 2010. Meiotic study of Acrida turrita (Linnaeus 1758), Paracinema luculenta Karsch 1896 and Morphacris fasciata (Thunberg 1815) (Orthoptera: Acrididae). International Journal of Biological and Chemical Sciences. 4 (6): 1914-1921

Stace, CA. 1980. Plant taxonomy and biosystematics. Edward Arnold Publishers Ltd. London

Steel, RGD. Torrie, JH. 1981. Principles and procedures of statistics. A biometrical approach. ( $2^{\text {nd }}$ edn). McGrawhill International Book Company, London.

White MJD. 1973. Animal cytology and Evolution. $3^{\text {rd }}$ edition. Cambridge Univsersity Press. 961 pp.

Williams GO. Ogunbiyi BI. 1995. Chromosome morphology and meiosis in Zonocerus. variegatus L. (Orthoptera: Pyrgomorphidae). Cytologia, 60: 111- 116. 